Classification

Physics Abstracts

$75.40-76.90-76.80$

\title{
Remarks on the study of spin glass dynamics by Mössbauer and muon techniques
}

\author{
F. Hartmann-Boutron \\ Laboratoire de Spectrométrie Physique (*), USMG, BP 53 X, 38041 Grenoble Cedex, France
}

(Reçu le 30 juillet 1982, accepté le 29 octobre 1982)

\begin{abstract}
Résumé. - Du fait de leurs échelles de temps différentes, la spectroscopie Mössbauer et la précession des muons positifs fournissent des renseignements complémentaires sur les verres de spin. Dans les verres de spin classiques, des expériences «longitudinales " en champ nul sur les muons ont été interprétées en termes de ralentissement de fluctuations de grande amplitude; nous montrons que ceci conduit à des temps caractéristiques au voisinage de $T_{\mathrm{G}}$ qui ne paraissent pas compatibles avec les résultats Mössbauer.
\end{abstract}

\begin{abstract}
Because of their different time scales, Mössbauer spectroscopy and $\mu^{+}$SR give complementary information on spin glasses. We show that for standard spin glasses in zero field, the interpretation of longitudinal muon experiments in terms of a slowing down of large amplitude fluctuations, leads to characteristic times around $T_{\mathrm{G}}$ which do not seem compatible with Mössbauer results $\left({ }^{* *}\right)$.
\end{abstract}

There is presently some uncertainty about the exact nature of the spin glass transition. Is it similar to a true phase transition (assumption (A)) or is it due to a progressive slowing down of large amplitude fluctuations of magnetic clusters (assumption (B)) ? Information about the dynamics of spin glasses can be obtained by various methods, among which Mössbauer and muon techniques play a central role. However the interpretation of the results which they provide requires some care : the aim of the present paper is to draw attention on a few important points.

1. Dynamics and static effects in Mössbauer spectra. - 1.1 DyNAMIC EFFECTs. - We only consider here the Mössbauer effect of ${ }^{57} \mathrm{Fe}\left(I=3 / 2 \rightarrow I_{\mathrm{g}}=1 / 2, E_{\gamma}=14.4 \mathrm{keV}\right)$. It is well known

(*) Laboratorre associé au C.N.R.S. (LA 08).

$(* *)$ The subsequent text is an expanded version of a communication $(8 \mathrm{G} 9)$ presented to the International Conference on Magnetism, Kyoto (1982). 
that when the Fe nucleus is submitted to a static hyperfine field $\mathbf{H}$, the Mössbauer spectrum of a powder is composed of six lines : a, $\mathrm{a}^{\prime}$ (external), b, $\mathrm{b}^{\prime}$ (median), c, $\mathrm{c}^{\prime}$ (internal) with relative intensities 3, 2, 1. What happens if $\mathbf{H}$ begins jumping between two opposite and equally probable values $H_{z}= \pm H$ ? At each jump, lines aa' exchange their frequencies and similarly for bb', $\mathrm{cc}^{\prime}$. If the jumping rate $\Omega=1 / \tau_{\mathrm{c}}$ is small compared to the Zeeman splitting the spectrum is unchanged, being the superposition of two identical spectra. If $\Omega$ increases, lines cc' $^{\prime}$ broaden and collapse, followed by $\mathrm{bb}^{\prime}$ and lastly by $\mathrm{aa}^{\prime}$. At the same time a line begins to grow at the centre of the spectrum, and for a fast jumping rate the spectrum reduces to this unique line (extreme narrowing limit). Computed spectra based on this model may be found in reference [1] : the intermediate zone corresponds to values of $\tau_{c}$ comparable to the hyperfine circular frequencies, i.e. : $\tau_{\mathrm{c}} \sim 5 \times 10^{-9} \mathrm{~s}-10^{-8} \mathrm{~s}$. The same qualitative evolution is observed for a fluctuating $\mathbf{H}$ of constant length which reorients randomly in any direction in space [2].

On the contrary let us now assume that $\mathbf{H}$ jumps between several values $H_{z}=H_{1}, H_{2}, H_{3}, \ldots$ which are unequal, with unequal probabilities $p_{1}, p_{2}, p_{3}$. For small jumping rates the spectrum is the sum of several different Zeeman spectra associated with $H_{1}, H_{2}, H_{3} \ldots$ Then as $\Omega$ increases the spectrum broadens and collapses and finally, in the very fast relaxation limit, it consists, not of a single line, but of an unbroadened Zeeman spectrum corresponding to the average field $\langle H\rangle=p_{1} H_{1}+p_{2} H_{2}+p_{3} H_{3} \ldots$ This is what Mössbauerists call an " effective field situation $\gg$.

Therefore an unbroadened Zeeman spectrum may correspond, either to a field jumping slowly between equal values, or to a field jumping rapidly between unequal values (or alternatively to $\mathbf{H}=\langle\mathbf{H}\rangle+\delta \mathbf{H}(t)$, where the fluctuations $\delta \mathbf{H}(t)$ are fast compared to the Larmor period associated with the average value $\langle\mathbf{H}\rangle$ ). A typical example of an effective field situation is encountered in standard magnets, where the hyperfine field is proportional to the magnetic moment : $\mathbf{H}_{n}=A\langle\mathbf{S}\rangle$ (in this case $\delta \mathbf{H}(t) \equiv A \delta \mathbf{S}(t)$ corresponds to spin wave modulations, whose frequencies are much higher than hyperfine frequencies). The relation $\mathbf{H}_{n}=A\langle\mathbf{S}\rangle$ is obeyed even very near $T_{N}$ [3] : comparison with $\mathrm{NMR}$ results in $\mathrm{MnF}_{2}$ [4] indeed suggests that for ${ }^{57} \mathrm{Fe}$, critical effects must be smaller than the Mössbauer linewidth, due to the smallness of hyperfine coupling constants.

Finally it should be noted that for a fluctuating field whose jumping rate varies from slow to fast with temperature (as in superparamagnets '[5]), the aspect of the ME spectra changes drastically on a relatively small temperature interval.

1.2 STATIC EFFECTS. - Because the Mössbauer transition takes place between nuclear states with different gyromagnetic factors, lines $\mathrm{cc}^{\prime}, \mathrm{bb}^{\prime}, \mathrm{aa}^{\prime}$ also correspond to different gyromagnetic ratios $\left(\left|\gamma_{\mathrm{cc}^{\prime}}\right|<\left|\gamma_{\mathrm{bb}^{\prime}}\right|<\left|\gamma_{\mathbf{a a}^{\prime}}\right|\right)\left({ }^{1}\right)$. Therefore, if the nuclei of the sample are submitted to an inhomogeneous static hyperfine field, all ME lines will be inhomogeneously broadened, but with increasing widths from $\mathrm{cc}^{\prime}$ (internal lines) tọ $\mathrm{aa}^{\prime}$ (external lines). Such spectra are observed, for example, in amorphous magnets.

1.3 SITUATION IN STANDARD SPIN GLASSES. - It should first be noticed that in non very dilute spin glasses, Mössbauer spectra above the "spin glasses transition " are often perturbed by quadrupole effects (associated with pairs of neighbouring atoms) which should not be interpreted as " short range order". When these effects do not exist ( $\mathrm{RhFe})$ [6] or are eliminated by dilution (AuFe) [7], above $T_{\mathrm{G}}$ the ME spectrum (Fig. 1) consists of a single line which broadens as $T$ decreases. It then splits into two wide wings, two small lines appear near the centre and progressively a full Zeeman structure develops. The fact that lines cc' $^{\prime}$ appear first and also that in

(') In $\mathrm{mm} / \mathrm{s}$ per $100 \mathrm{kG}:\left|\gamma_{\mathrm{a}} / 2 \pi\right|=1.61,\left|\gamma_{\mathrm{b}} / 2 \pi\right|=0.93,\left|\gamma_{\mathrm{c}} / 2 \pi\right|=0.25 ; 1 \mathrm{~mm} / \mathrm{s} \leftrightarrow 11.6 \mathrm{MHz}$. 


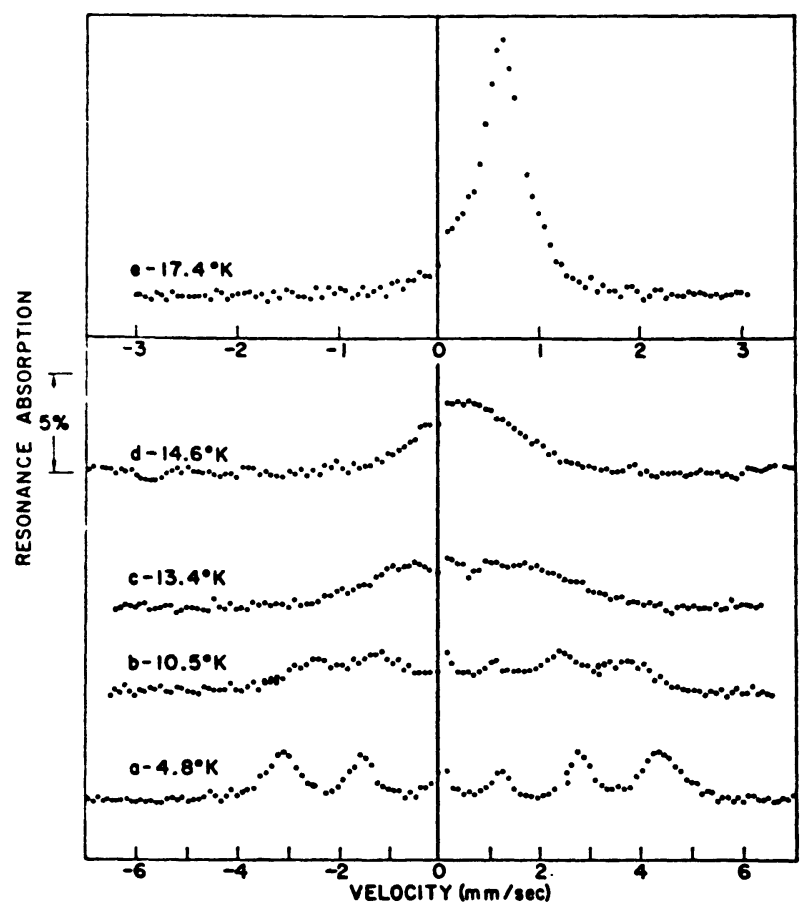

Fig. 1. $-{ }^{57} \mathrm{Fe}$ Mössbauer spectra of $\underline{\mathrm{Au}} 1.7 \% \mathrm{Fe}$ (estimated $T_{\mathrm{G}}=14.8 \pm 0.5 \mathrm{~K}$ ). The small shoulder on the left in the $17.4 \mathrm{~K}$ spectrum is part of a quadrupole doublet associated with residual pair effects. After Violet and Borg, Ref. [7].

the full spectrum the linewidths increase from $\mathrm{cc}^{\prime}$ to $\mathrm{aa}^{\prime}$ is strongly suggestive of an " effective field situation " (case (A)) in the presence of inhomogeneous broadening (on the contrary, for slowing down fluctuations (case (B)) $\mathrm{aa}^{\prime}$ would appear first and $\mathrm{cc}^{\prime}$ last, the aspect of the spectrum would be different and would change rapidly). When $T$ continues decreasing, the average Zeeman splitting grows (as in a ferromagnet) while the line broadening diminishes. This may be interpreted in the following way : because of disorder, the different magnetic atoms $i$ are submitted to different exchange fields $\mathbf{H}_{\mathrm{ex}}(i)$, whence a distribution of the lengths $|\langle\mathbf{S}(i)\rangle|$ of their average spins $\langle\mathbf{S}(i)\rangle$ and a distribution of effective hyperfine fields $\left(\left|\mathbf{H}_{n}(i)\right| \propto|\langle\mathbf{S}(i)\rangle|\right)$. When $T$ decreases, $\left\langle\left|\left\langle\mathbf{H}_{\mathrm{ex}}(i)\right\rangle\right|\right\rangle$ (averaged over $i$ ) increases and so do $\langle|\langle\mathbf{S}(i)\rangle|\rangle$ and $\left\langle\left|\mathbf{H}_{n}(i)\right|\right\rangle$. However when $T \rightarrow 0$, because of saturation $\left|\left\langle\mathbf{S}_{i}\right\rangle\right| \rightarrow S$ whatever $\mid\left\langle\mathbf{H}_{\mathrm{ex}}(i)\right\rangle$, whence the line narrowing. Mössbauer experiments therefore seem to support assumption (A). Note however that in more complicated systems (amorphous or ternary compounds) it may not be possible to choose between static and dynamic interpretations.

2. Dynamic and static effects in muon techniques. - Let us recall that muons have a lifetime $\tau_{\mu}=2.2 \times 10^{-6} \mathrm{~s}$ and a spin $s_{\mu}=1 / 2$, with a gyromagnetic ratio $\gamma_{\mu} / 2 \pi=13 \mathrm{kHz} / \mathrm{G}$.

In solids there are two types of muon $\mu^{+}$experiments :

- transverse precession experiments in an applied field $H_{0}$ [8], which are similar to NMR free precession experiments and measure a quantity $G_{x}(t) \propto \overline{s_{x}^{\mu}(0) s_{x}^{\mu}(t)}$,

- longitudinal experiments, with or without an applied field $H_{0}$ [9], which measure $G_{z}(t) \propto \overline{s_{z}^{\mu}(0) s_{z}^{\mu}(t)}$.

In spin glasses both techniques are used, with an observation time of the order $1 \mu$ s to several $\mu \mathrm{s}$ and a resolution of the order $50 \mathrm{~ns}$ to $100 \mathrm{~ns}$. 
At low $T$, muons occupy fixed interstitial positions $\alpha$ and, in dilute spin glasses, they are submitted to a field $\mathbf{H}(\alpha)$ approximately given by [10] : $\mathbf{H}(\alpha)=\mathbf{H}_{\|}+\mathbf{H}_{\text {dip }}(\alpha)$, in which $\mathbf{H}_{\|} \simeq \mathbf{H}_{0}$ and $\mathbf{H}_{\mathrm{dip}}(\alpha)$ is the field with dipolar symmetry created at the muon $\alpha$ by the magnetic moments of its neighbours $i_{\alpha}$ in a Lorentz sphere (typically, at concentrations $c \sim 1 \%,\left|\mathbf{H}_{\mathrm{dip}}(\alpha)\right| \lesssim 100 \mathrm{Oe}$ ). Both length and orientation of $\mathbf{H}_{\text {dip }}(\alpha)$ depend on $\alpha$. If the spin glass is composed of magnetic clusters with large amplitude fluctuations (assumption (B)), then the length of $\mathbf{H}_{\text {dip }}(\alpha)$ should correspond to the full magnetic moments and be temperature independent, while its orientation should depend on time with some characteristic time $\tau_{\mathrm{c}}$ (dipole-dipole anisotropy is neglected for simplicity). On the contrary, within assumption (A) (phase transition),

$$
\mathbf{H}_{\mathrm{dip}}(\alpha)=\left\langle\mathbf{H}_{\mathrm{dip}}(\alpha)\right\rangle+\delta \mathbf{H}_{\mathrm{dip}}(\alpha, t),
$$

in which the time independent average value $\left\langle\mathbf{H}_{\text {dip }}(\alpha)\right\rangle$ is related to the $\left\langle\mathbf{S}\left(i_{\alpha}\right)\right\rangle$ 's of the neighbours, while $\delta \mathbf{H}_{\mathrm{dip}}(\alpha, t)$ is associated with their rapid fluctuations $\delta \mathbf{S}\left(i_{\alpha}, t\right)$ (spin wave excitations, etc.) which do not influence the muon (" effective field situation »). Because of disorder $\mathbf{H}_{\text {dip }}(\alpha)$ varies with $\alpha$, but the average over $\alpha$ of its length, $\left\langle\left|\left\langle\mathbf{H}_{\text {dip }}(\alpha)\right\rangle\right|\right\rangle$, should be proportional to the average magnetization $\langle|\langle\mathbf{S}(i)\rangle|\rangle$ and increase with decreasing temperature. Both with assumptions (A) and (B), the distribution of lengths associated with positional and directional disorders should be a Gaussian in the concentrated case and a Lorentzian in the dilute case [11], characterized by their widths $\Delta$.

Therefore two possible explanations should be examined for interpreting muon depolarization in spin glasses :

- either a time independent average $\left\langle\mathbf{H}_{\text {dip }}(\alpha)\right\rangle$ with a distribution of width

$$
\Delta(T) \propto\langle|\langle\mathbf{S}(T)\rangle|\rangle,
$$

which increases when $T$ decreases (assumption (A)),

- or a fluctuating $\mathbf{H}_{\text {dip }}(\alpha)$ with a variable jumping rate $v(T) \equiv 1 / \tau_{\mathrm{c}}(T)$ that decreases with temperature, but with a diștribution of constant (maximum) width $\Delta \sim S$ (assumption (B)).

With their present accuracy, transverse muon experiments are not able to distinguish between static effects (A) and dynamic damping (B). Although they are also sensitive to inhomogeneous broadening, longitudinal muon experiments in zero field have been asserted to support the dynamic assumption (B) but this deserves careful examination.

First on the theoretical side $[9 a, 12,13]$ : in the static limit, both Gaussian (Fig. 2a) and Lorentzian (Fig. $2 b$ ) distributions lead to decreasing $G_{z}(t)$ 's (with different departures), which go through a minimum when $t \Delta \sim 1.8$ before tending to $1 / 3$ as $t \rightarrow \infty$; on the contrary in the fast relaxation limit, only with the Gaussian distribution does one recover the extreme narrowing effect (damped $G_{z}(t)$ with a damping constant $\sim \Delta^{2} / v \sim \Delta^{2} \tau_{\mathrm{c}}$ ). Various recipes had to be devised in order to

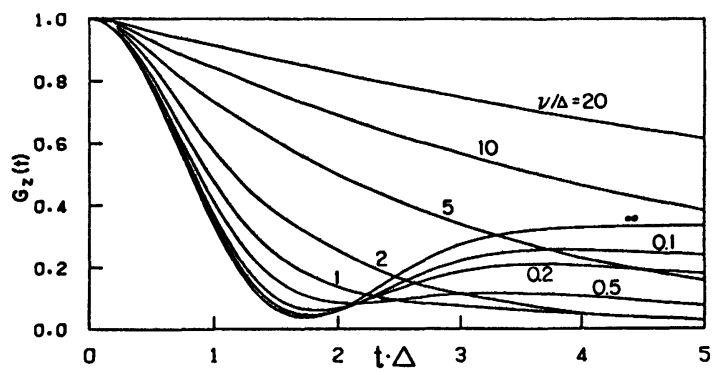

Fig. 2a. - Computed longitudinal depolarization function $G_{z}(t, \Delta, v)$, as a function of $(\Delta t)$, for a fluctuating Gaussian field with jumping rate $v$. The static case corresponds to $v / \Delta=0$. After Hayano et al. Ref. [9a]. 


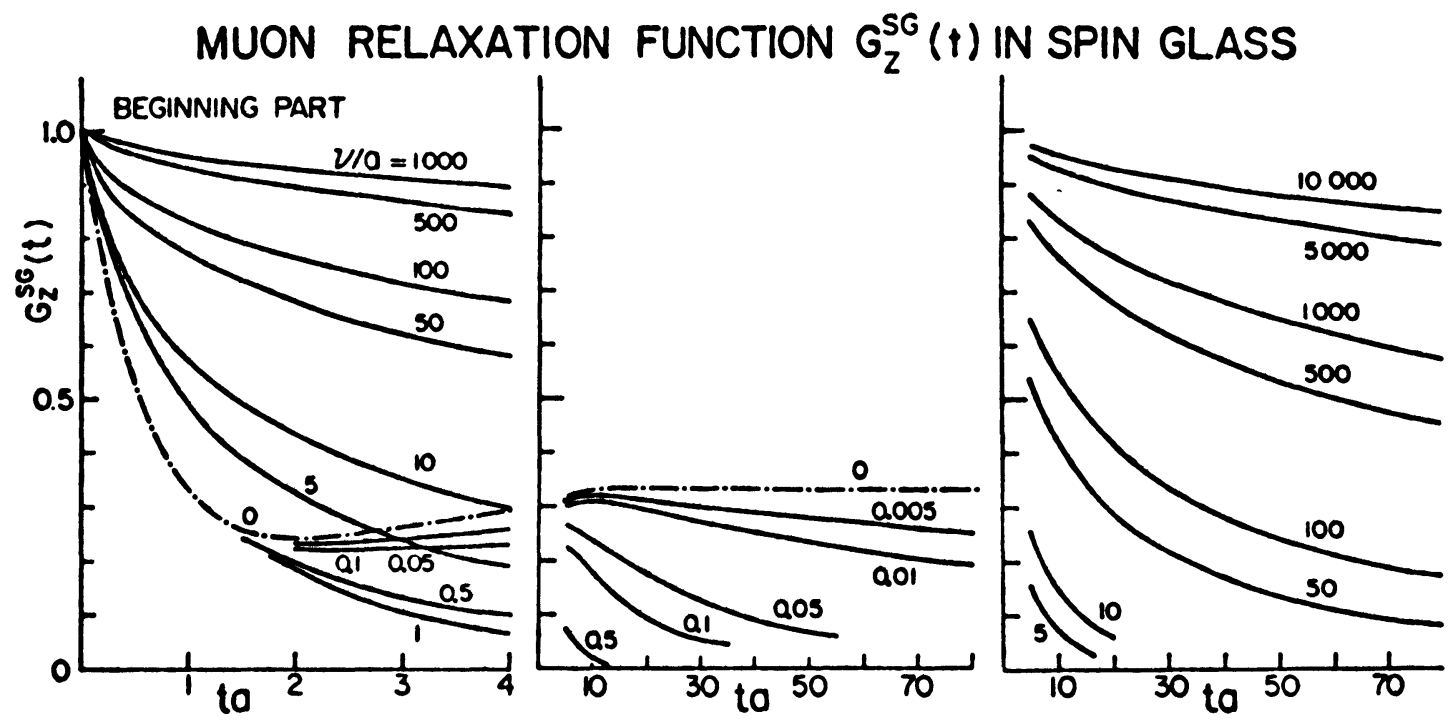

Fig. 2b. - Computed longitudinal depolarization functions $G_{z}^{\mathrm{SG}}(t, a, v)$ as a function of $(a t)$ in the Lorentzian dilute case, as obtained by Uemura et al., Refs. $[9 b, 9 c]$. This is the result of a calculation in which the averages over the orientations of the spins and the positions of the spins are carried out separately. The static case is represented by the dot dashed line. In the notation of the present paper, $a$ should be replaced by $\Delta$.

obtain the narrowing effect even in the Lorentzian (dilute) case [9d,13]. When this is achieved it appears that the sets of curves $G_{z}(t)$ corresponding to assumptions (A) $(\Delta \uparrow, v=0)\left({ }^{2}\right)$ and (B) (fixed $\Delta, v \downarrow$ ) look very similar except at long $t$. With (A), $G_{z}(t)$ goes through a minimum when $t \Delta \sim 2$ and then tends to $1 / 3$ when $t \rightarrow \infty$. With (B), at low $v / \Delta, G_{z}(t)$ exhibits first a minimum and then a hump before tending to zero; at high $v / \Delta, G_{z}(t)$ goes monotonically to zero with decreasing damping.

Now when one looks at the experimental curves for $G_{z}(t)$ in standard spin glasses below $T_{\mathrm{G}}[9 d]$ and figure 3, their present statistics do not seem good enough to clearly choose between (A) and (B) (no unquestionable minima and, all the more, humps, can be seen). However most authors

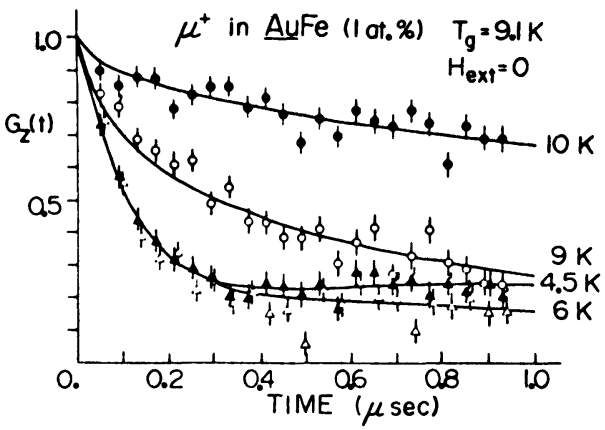

Fig. 3. - Experimental depolarization function in Au $1 \%$ Fe. After Ref. [9b].

${ }^{2}$ ) In the literature there is no set of curves corresponding to case (A), because $G_{z}$ is plotted as a function of the reduced variable $\Delta t$ (see Figs. $2 a, 2 b$ ). 
have interpreted their experiments on the basis of assumption (B). In this way they find a rapid slowing down $(v \downarrow)$ when $T$ decreases below $T_{\mathrm{G}}$, with a characteristic fluctuation time $\tau_{\mathrm{c}}$ around $T_{\mathrm{G}}$ of the order $\tau_{\mathrm{c}} \sim 10^{-8} \mathrm{~s}$ in CuMn, AgMn and AuFe ([14] Fig. 2). But if this happens to be true, $\tau_{\mathrm{c}}$ being comparable to the usual Mössbauer hyperfine periods, ME spectra of AuFe at $T_{\mathrm{G}}$ should rapidly develop a full hyperfine structure (similar to what is observed in superparamagnets). This does not occur at all and this fact, together with the satisfactory interpretation of ME spectra in terms of inhomogeneous broadening is not in favour of assumption (B). With assumption (A) the increasing damping below $T_{\mathrm{G}}$ is simply accounted for by the thermal variation of

$$
\Delta(T) \propto\left\langle\left|\left\langle\mathbf{S}_{i}\right\rangle\right|\right\rangle ;
$$

obiously this does not eliminate the possibility of a critical slowing down of certain excitation modes near $T_{\mathrm{G}}$ as in standard magnets, but information on the excitation spectrum at low frequency is lacking.

To summarize, in the present status of both theory and experiment, muon damping in standard spin glasses in zero field can be attributed either (A) to an increasing, non fluctuating, average $\left\langle\mathbf{H}_{\text {dip }}(\alpha)\right\rangle$, the deviations of $\mathbf{H}_{\text {dip }}(\alpha)$ with respect to this average being too fast to influence the muon or (B) to a fluctuating $\mathbf{H}_{\mathrm{dip}}(\alpha)$ of fixed (maximum) length whose fluctuations slow down. The fluctuating time at $T_{\mathrm{G}}$ derived with assumption (B) does not seen to be compatible with the Mössbauer spectra of ${ }^{57} \mathrm{Fe}$, which are better described in terms of an increasing effective field, together with a large inhomogeneous broadening, in agreement with assumption (A).

In fact with muons the only safe way for discriminating between dynamic and static effects is to perform longitudinal experiments in the presence of an external decoupling field $H_{0} \gg\left|H_{\mathrm{dip}}^{\max }\right|$ [15] : this field suppresses static damping, however it also reduces dynamic damping ([15], Eq. (11)) and probably perturbs the spin glasses.

A final conclusion is that the situation in spin glasses is far from being understood, as shown by the generalized use of Log-Log plots in the literature and by the comparison of informations derived from different techniques ([17], Fig. 1).

Acknowledgments. - I am indebted to Dr. P. Monod for calling my attention to this problem.

Note added in proof. - Uemura et al. have recently performed longitudinal $\mu^{+}$experiments on $\mathrm{CuMn}$ in the presence of an applied field larger than the dipolar field. A strong reduction in the damping of $G_{z}(t)$ is observed immediately below $T_{\mathrm{G}}$; this has been attributed to the emergence of a quasi-static component of $\mathbf{S}$, giving rise to decoupling effects similar to those expected in the presence of an average $\Delta(T)$ under assumption (A). However an additional fluctuating part $\delta \mathbf{S}(t)$ with a fairly long characteristic time $\tau_{\mathrm{c}} \sim 10^{-8}-10^{-9} \mathrm{~s}$ is still introduced into the theory (see also $[14,16])$. More details about these experiments and about the various models elaborated in reference [9] can be found in Y. J. Uemura's thesis, as well as in the communications $3 \mathrm{G} 14$ (Uemura et al.) and 7 E 2(Yamazaki et al.) to ICM $82 \mathrm{Kyoto}$. I am greatly indebted to Dr. Uemura for communicating his thesis.

\section{References}

[1] Wickman, H. H., Mössbauer Effect Methodology, vol. 2, p. 39, I. J. Gruverman Ed. (Plenum Press) 1966. See Fig. 6, p. 55.

[2] Dattagupta, S., Hyp. Int. 11 (1981) 77. See Fig. 5, p. 105.

[3] Wertheim, G. K., Mössbauer Effect Methodology, vol. 4, p. 159, I. J. Gruverman Ed. (Plenum Press) 1968. See Fig. 2, p. 164 ; Figs. 9-10, p. 174-175.

[4] Heller, P., in Critical Phenomena, Proc. of a Conference, Washington (1965) (Ed. by M. S. Green, J. V. Sangers, NBS Misc) 1973. 
[5] VAN Der KraAN, A. M., J. Physique Coll. 32 (1971) C1-1034, ICM Grenoble. In real superparamagnets there is a distribution of $\Omega$ due to different grain sizes.

[6] Window, B. et al., J. Phys. C 3 (1970) 2156. See Fig. 3.

[7] Violet, C. E. et al., Phys. Rev. 149 (1966) 540. See Fig. 2.

[8] Murnick, D. E. et al., Phys. Rev. Lett. 36 (1976) 100.

[9] a) Hayano, R. S. et al., Phys. Rev. B 20 (1979) 850 ;

b) Uemura, Y. J. et al., Phys. Rev. Lett. 45 (1980) 583 ;

c) Uemura, Y. J., Solid State Commun. 36 (1980) 369;

d) Uemura, Y. J., Hyp. Int. 8 (1981) 739.

[10] Meier, P. F., Hyp. Int. 8 (1981) 591.

[11] Walstedt, R. E. et al., Phys. Rev. B 9 (1974) 4857.

[12] Kubo, R., Hyp. Int. 8 (1981) 731.

[13] LeON, M., Hyp. Int. 8 (1981) 781.

[14] Heffner, R. H. et al., J. Appl. Phys. 53 (1982) 2174.

[15] Yamazaki, T., Hyp. Int. 6 (1979) 115.

[16] EMMERICH, K., Hyp. Int. 12 (1982) 59.

[17] MaC Laughlin, D. E., Hyp. Int. 8 (1981) 749. 